International Journal of Pure and Applied Mathematics

Volume 83 No. 2 2013, 271-278

ISSN: $1311-8080$ (printed version); ISSN: 1314-3395 (on-line version)

url: http://www.ijpam.eu

doi: http://dx.doi.org/10.12732/ijpam.v83i2.6

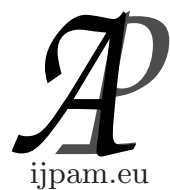

\title{
ON A QUARTER SYMMETRIC NON-METRIC CONNECTION IN A KENMOTSU MANIFOLDS
}

\author{
Amit Prakash ${ }^{1}$, Vijay Kumar Pandey ${ }^{2} \S$ \\ ${ }^{1}$ Department of Mathematics \\ Allenhouse Institute of Technology \\ Rooma, Kanpur, U.P. INDIA \\ ${ }^{2}$ Department of Mathematics \\ Integral University \\ Kursi Road, Lucknow, U.P. INDIA
}

\begin{abstract}
In this paper we study a quarter-symmetric non-metric connection in a Kenmotsu manifold.
\end{abstract}

AMS Subject Classification: 58D17, 57R15

Key Words: Kenmotsu manifolds, quarter-symmetric non-metric connection

\section{Introduction}

In 1975, Golab [11] introduced the notion of quarter-symmetric connection in a Riemannian manifold with affine connection. This was further developed by Jun, De and Pathak [4], Yano and Imai [6], Rastogi [13], [14], Mishra and Pandey [8], Mukhopadhyay, Roy and Barua [12], Biswas and De [10], Sengupta and Biswas [3], Singh and Pandey [9] and many other geometers.

In this paper we have studied a quarter-symmetric non-metric connection in a Kenmotsu manifold, as introduced by Prakash and Narain [1]. The curvature tensor and the Ricci tensor of the quarter-symmetric non-metric connection is found. A necessary and sufficient condition for the Ricci tensor of $\bar{D}$ to be skewsymmetric under certain conditions. A necessary and sufficient condition for

Received: September 8, 2012

(C) 2013 Academic Publications, Ltd.

$\S_{\text {Correspondence author }}$ url: www.acadpubl.eu 
the projective Ricci tensor of $\bar{D}$ to be skew-symmetric under certain condition. We also find the necessary and sufficient condition for the Einstein manifold of the connection $\mathrm{D}$ is equal to the Einstein manifold of the connection $\bar{D}$ under certain condition.

\section{Preliminaries}

Let $M^{n}$ be an $n$ - dimensional almost contact Riemannian manifold with an almost contact metric structure $(\phi, \xi, \eta, g)$, where $\phi$ is a $(1,1)$-tensor field, $\xi$ is a vector field, $\eta$ is a 1 -form and $g$ is the Riemannian metric, which satisfy (see $[2],[5])$

$$
\begin{gathered}
\phi^{2} X=-X+\eta(X) \xi, \\
\eta(\xi)=1, \\
g(\phi X, \phi Y)=g(X, Y)-\eta(X) \eta(Y), \\
g(X, \xi)=\eta(X), \\
\left(D_{X} \phi\right)(Y)=-g(X, \phi Y) \xi-\eta(Y) \phi X, \\
D_{X} \xi=X-\eta(X) \xi,
\end{gathered}
$$

for any vector fields $\mathrm{X}$ and $\mathrm{Y}$, where $D$ denotes covariant differentiation with respect to g.

Also, in a Kenmotsu manifold $M^{n}$ with structure $(\phi, \xi, \eta, g)$ the following relation hold: (see [4])

$$
\begin{gathered}
\text { (a) } \quad \phi \xi=0 \quad(b) \quad \eta(\phi X)=0 \quad(c) \quad \operatorname{rank}(\phi)=(n-1) . \\
\left(D_{X} \eta\right)(Y)=g(X, Y)-\eta(X) \eta(Y), \\
R(X, Y, Z, \xi)=g(X, Z) \eta(Y)-g(Y, Z) \eta(X),
\end{gathered}
$$

and

$$
S(X, \xi)=-(n-1) \eta(X),
$$

where $R$ and $S$ denotes curvature tensor of type $(0,4)$ and the Ricci tensor of type $(0,2)$ respectively.

A quarter-symmetric non-metric connection $\bar{D}$ in an LP-Sasakian manifold is defined by (Prakash and Narain [1])

$$
\bar{D}_{X} Y=D_{X} Y+\eta(Y) \phi X+a(X) \phi Y .
$$




\section{The Curvature Tensor of a Kenmotsu Manifold with Respect to the Quarter-Symmetric Non-Metric Connection $\overline{\mathrm{D}}$}

Let $\bar{R}$ and $R$ be the curvature tensor of the connection $\bar{D}$ and $D$ respectively, then

$$
\bar{R}(X, Y) Z=\bar{D}_{X} \bar{D}_{Y} Z-\bar{D}_{Y} \bar{D}_{X} Z-\bar{D}_{[X, Y]} Z .
$$

From (1.11) and (3.1), we get

$$
\begin{gathered}
\bar{R}(X, Y) Z=\bar{D}_{X}\left(D_{Y} Z+\eta(Z) \phi Y+a(Y) \phi Z\right)-\bar{D}_{Y}\left(D_{X} Z+\eta(Z) \phi X+a(X) \phi Z\right) \\
-D_{[X, Y]} Z-\eta(Z) \phi([X, Y])-a([X, Y]) \phi Z
\end{gathered}
$$

Using (1.2), (1.5), (1.6) and (1.8) in (3.2), we get

$$
\begin{aligned}
& \bar{R}(X, Y) Z \\
& =R(X, Y) Z+g(X, Z) \phi Y-g(Y, Z) \phi X+2 g(\phi X, Y) \eta(Z) \xi-g(\phi Y, Z) a(X) \xi \\
& +g(\phi X, Z) a(Y) \xi+a(X) \eta(Z) \phi Y-a(Y) \eta(Z) \phi X+a(Y) \eta(Z) X-a(X) \eta(Z) Y \\
& +a(X) \eta(Y) \eta(Z) \xi-a(Y) \eta(X) \eta(Z) \xi+d a(X, Y) \phi Z, \quad(3.3)
\end{aligned}
$$

where

$$
R(X, Y) Z=D_{X} D_{Y} Z-D_{Y} D_{X} Z-D_{[X, Y]} Z \text { is the curvature }
$$
tensor of $\mathrm{D}$ with respect to the Riemannian connection.

Contracting (3.3), we find

$$
\begin{aligned}
\bar{S}(Y, Z) & =S(Y, Z)+[1-a(\xi)] g(\phi Y, Z)+(n-2) a(Y) \eta(Z) \\
& +a(\xi) \eta(Y) \eta(Z)+a(\phi Y) \eta(Z)+d a(\phi Z, Y) .
\end{aligned}
$$

and

$$
\bar{r}=r+(n-1) a(\xi)+\lambda,
$$

where $\bar{S}$ and $\bar{r}$ are the Ricci tensor and scalar curvature with respect to $\bar{D}$ and

$$
\lambda=\operatorname{trace} d a(\phi Z, Y) .
$$

Hence, we can state the following theorem :

Theorem 3.1. The curvature tensor $\bar{R}(X, Y) Z$, the Ricci tensor $\bar{S}(Y, Z)$ and the scalar curvature $\bar{r}$ of a Kenmotsu manifold with respect to quartersymmetric non-metric connection is given by (3.3), (3.4) and (3.5) respectively.

Let us assume that $\bar{R}(X, Y) Z=0$ in (3.3) and contracting, we get

$$
S(Y, Z)=-[1-a(\xi)] g(\phi Y, Z)-(n-2) a(Y) \eta(Z)
$$




$$
-a(\xi) \eta(Y) \eta(Z)-a(\phi Y) \eta(Z)-d a(\phi Z, Y) .
$$

which gives

$$
r=-(n-1) a(\xi)-\lambda .
$$

Hence, we can state the following theorem.

Theorem 3.2. If a Kenmotsu manifold $M^{n}$ admits a quarter-symmetric non-metric connection whose curvature tensor vanishes, then the scalar curvature $r$ is given by $r=-(n-1) a(\xi)-\lambda$.

\section{Skew-Symmetric Condition of Ricci Tensor of $\bar{D}$ in a Kenmotsu Manifold}

From (3.4), we have

$$
\begin{aligned}
\bar{S}(Z, Y) & =S(Z, Y)+[1-a(\xi)] g(\phi Z, Y)+(n-2) a(Z) \eta(Y) \\
& +a(\xi) \eta(Y) \eta(Z)+a(\phi Z) \eta(Y)+d a(\phi Y, Z) .
\end{aligned}
$$

From (3.4) and (4.1), we have

$$
\begin{gathered}
\bar{S}(Y, Z)+\bar{S}(Z, Y)=2 S(Y, Z)+(n-2) a(Y) \eta(Z)+a(Z) \eta(Y) \\
+2 a(\xi) \eta(Y) \eta(Z)+a(\phi Z) \eta(Y)+a(\phi Y) \eta(Z)+d a(\phi Y, Z)+d a(\phi Z, Y) .
\end{gathered}
$$

If $\bar{S}(Y, Z)$ is skew-symmetric then the left hand side of (4.2) vanishes, and we get

$$
\begin{gathered}
S(Y, Z)=-a(\xi) \eta(Y) \eta(Z)-\frac{(n-2)}{2}[a(Y) \eta(Z)+a(Z) \eta(Y)] \\
-\frac{1}{2}[a(\phi Z) \eta(Y)+a(\phi Y) \eta(Z)+d a(\phi Y, Z)+d a(\phi Z, Y)] .
\end{gathered}
$$

Moreover if $S(Y, Z)$ is given by (4.3), then from (4.2), we get

$$
\bar{S}(Y, Z)+\bar{S}(Z, Y)=0 .
$$

Hence, we can state the following theorem :

Theorem 4.1. If a Kenmotsu manifold admits a quarter-symmetric nonmetric connection $\bar{D}$ then a necessary and sufficient condition for the Ricci tensor of $\bar{D}$ to be skew-symmetric is that the Ricci tensor of the Levi-Civita connection $D$ is given by (4.3). 


\section{Skew-Symmetric Properties of Projective Ricci Tensor with Respect to Quarter-Symmetric Non-Metric Connection $\bar{D}$ in a Kenmotsu Manifold}

Projective Ricci tensor in a Riemannian manifold is defined by (Chaki and Saha [7]) as follows

$$
P(X, Y)=\frac{n}{(n-1)}\left[S(X, Y)-\frac{r}{n} g(X, Y)\right] .
$$

Analogous to this definition, we define projective Ricci tensor with respect to quarter-symmetric non-metric connection $\bar{D}$, given by

$$
\bar{P}(X, Y)=\frac{n}{(n-1)}\left[\bar{S}(X, Y)-\frac{\bar{r}}{n} g(X, Y)\right] .
$$

From (3.4), (3.5) and (5.2), we have

$$
\begin{aligned}
\bar{P}(X, Y)= & \frac{n}{(n-1)}[S(X, Y)+\{1-a(\xi)\} g(\phi X, Y) \\
& +(n-2) a(X) \eta(Y)+a(\xi) \eta(X) \eta(Y) \\
& +a(\phi X) \eta(Y)+d a(\phi Y, X) \\
& \left.-\left\{\frac{r+(n-1) a(\xi)+\lambda}{n}\right\} g(X, Y)\right] .
\end{aligned}
$$

From (5.3), we have

$$
\begin{aligned}
\bar{P}(Y, X)= & \frac{n}{(n-1)}[S(Y, X)+\{1-a(\xi)\} g(\phi Y, X) \\
& +(n-2) a(Y) \eta(X)+a(\xi) \eta(X) \eta(Y) \\
& +a(\phi Y) \eta(X)+d a(\phi X, Y) \\
& \left.-\left\{\frac{r+(n-1) a(\xi)+\lambda}{n}\right\} g(Y, X)\right] .
\end{aligned}
$$

From equation (5.3) and (5.4), we have

$$
\begin{aligned}
\bar{P}(X, Y)+ & \bar{P}(Y, X)=\frac{n}{(n-1)}[2 S(X, Y)+(n-2)\{a(Y) \eta(X)+a(X) \eta(Y)\} \\
& +2 a(\xi) \eta(X) \eta(Y)-2\left\{\frac{r+(n-1) a(\xi)+\lambda}{n}\right\} g(X, Y) \\
& +a(\phi X) \eta(Y)+a(\phi Y) \eta(X)+d a(\phi X, Y)+d a(\phi Y, X)]
\end{aligned}
$$


If $\bar{P}(X, Y)$ is skew-symmetric then the left hand side of (5.5) vanishes, and we get

$$
\begin{gathered}
S(X, Y)=\left[-a(\xi) \eta(X) \eta(Y)-\frac{(n-2)}{2}\{a(Y) \eta(X)+a(X) \eta(Y)\}\right. \\
-\frac{1}{2}\{a(\phi X) \eta(Y)+a(\phi Y) \eta(X)+d a(\phi X, Y)+d a(\phi Y, X)\} \\
\left.+\left\{\frac{r+(n-1) a(\xi)+\lambda}{n}\right\} g(X, Y)\right] .
\end{gathered}
$$

Moreover if $S(X, Y)$ is given by (5.6), then from (5.5), we get

$$
\bar{P}(X, Y)+\bar{P}(Y, X)=0,
$$

i.e. Projective Ricci tensor of $\bar{D}$ is skew-symmetric.

Hence, we can state the following theorem :

Theorem 5.1. If a Kenmotsu manifold admits a quarter-symmetric nonmetric connection $\bar{D}$ then a necessary and sufficient condition for the Projective Ricci tensor of $\bar{D}$ to be skew-symmetric is that the Ricci tensor of the LeviCivita connection $D$ is given by (5.6).

\section{Einstein Manifold with Respect to Quarter-Symmetric Non-Metric Connection $\bar{D}$ in a Kenmotsu Manifold}

A Riemannian manifold $M^{n}$ is called an Einstein manifold with respect to Riemannian connection if

$$
S(X, Y)=\frac{r}{n} g(X, Y) .
$$

Analogous to this definition, we define Einstein manifold with respect to quarter-symmetric non-metric connection $\bar{D}$ by

$$
\bar{S}(X, Y)=\frac{\bar{r}}{n} g(X, Y) .
$$

From (3.4), (3.5) and (6.2), we have

$$
\begin{gathered}
\bar{S}(X, Y)-\frac{\bar{r}}{n} g(X, Y)=S(X, Y)-\frac{r}{n} g(X, Y)+\{1-a(\xi)\} g(\phi X, Y) \\
+(n-2) a(X) \eta(Y)+a(\xi) \eta(X) \eta(Y)+a(\phi X) \eta(Y)+d a(\phi Y, X)
\end{gathered}
$$




$$
-\left[\frac{(n-1) a(\xi)+\lambda}{n}\right] g(X, Y) .
$$

If

$$
\begin{gathered}
\{1-a(\xi)\} g(\phi X, Y)+d a(\phi Y, X)=\left[\frac{(n-1) a(\xi)+\lambda}{n}\right] g(X, Y) \\
-(n-2) a(X) \eta(Y)-a(\xi) \eta(X) \eta(Y)-a(\phi X) \eta(Y) .
\end{gathered}
$$

Then from (6.3), we get

$$
\bar{S}(X, Y)-\frac{\bar{r}}{n} g(X, Y)=S(X, Y)-\frac{r}{n} g(X, Y) .
$$

Hence, we can state the following theorem.

Theorem 6.1. In a Kenmotsu manifold $M^{n}$ with quarter-symmetric nonmetric connection if the relation (6.4) holds, then the manifold is an Einstein manifold for the Riemannian connection if and only if it is an Einstein manifold for the connection $\bar{D}$.

\section{References}

[1] A. Prakash, D. Narain, On a quarter-symmetric non-metric connection in a Lorentzian para-Sasakian manifolds, International Electronic Journal of Geometry, 4, No. 1 (2011), 129-137.

[2] D.E. Blair, Contact Manifolds in Riemannian Geometry, Lecture Notes in Math., 509, Springer Verlag.

[3] J. Sengupta, B. Biswas, Quarter-symmetric non-metric connection on a Sasakian manifold, Bull. Cal. Math. Soc., 95, No. 2 (2003), 169-176.

[4] J.B. Jun, U.C. De, G. Pathak, On Kenmotsu manifolds, J. Korean Math. Soc., 42, No. 3 (2005), 435.

[5] K. Kenmotsu, A class of almost contact Riemannian manifolds, Tohoku Math. J., 24 (1972), 93.

[6] K. Yano, T. Imai, Quarter-symmetric metric connections and their curvature tensors, Tensor, N.S., 38 (1982), 13-18.

[7] M.C. Chaki, S.K. Saha, On pseudo projective Ricci-symmetric manifolds, Bulg. Journ. Phys., 24 (1994). 
[8] R.S. Mishra, S.N. Pandey, On quarter-symmetric metric F-connections, Tensor, N.S. 34 (1980), 1-7.

[9] R.N. Singh, M.K. Pandey, On a type of quarter-symmetric non-metric connection in a Kenmotsu manifold, Bull. Cal. Math. Soc., 99, No. 4 (2007), 433-444.

[10] S.C. Biswas, U.C. De, Quarter-symmetric metric connection in an SPSasakian manifold, Common. Fac. Sci. Univ. Ank. Al., 46 (1997), 49.

[11] S. Golab, On semi-symmetric and quarter-symmetric linear connections, Tensor, N.S., 29 (1975), 249-254.

[12] S. Mukhopadhyay, A.K. Ray, B. Barua, Some properties of a quartersymmetric metric connection on a Riemannian manifold, Soochow J. of Math., 17, No. 2 (1991), 205.

[13] S.C. Rastogi, On quarter-symmetric metric connection, C.R. Acad. Sci., Bulgar, 31 (1978), 811-814.

[14] S.C. Rastogi, On quarter-symmetric metric connection, Tensor, N.S., 44 (1987), 133-141. 